\title{
Prospective study of body mass index and risk of sarcoidosis in US women
}

\author{
To the Editor:
}

Sarcoidosis is a systemic inflammatory disorder, characterised by the formation of immune granulomas that can occur in various organs, but typically affects the lungs [1]. Although sarcoidosis resolves spontaneously in many cases, about one-third of patients experience chronic disease associated with significant morbidity [2]

Although the exact causes of sarcoidosis remain unknown, it is thought to result from an exaggerated immune response to yet unidentified antigens in individuals with genetic susceptibility [1]. Geographical variations in sarcoidosis occurrence support the existence of environmental risk factors [3-5]. Research into the potential causes of sarcoidosis has focused on occupational and environmental exposures $[6,7]$, but lifestyle-related risk factors have not been as extensively examined.

The proinflammatory milieu of obesity appears to favour the development of other inflammation-related diseases, such as asthma [8]. Recently, a study of African-American women found that obesity was prospectively associated with a higher risk of incident sarcoidosis [9]. Black Americans are known to have an increased risk of sarcoidosis compared to white Americans, and risk factors may differ across these two populations. Although an association between obesity and sarcoidosis has also been suggested in two studies of predominantly white populations $[10,11]$, data were either cross-sectional or included limited information regarding weight change over time and potential confounders. In the current study, we aimed to investigate the prospective association of body mass index (BMI) and weight change with a 24-year incidence of sarcoidosis in a large cohort of female nurses in the U.S.

The Nurses' Health Study II (NHSII) began in 1989, when 116430 female registered nurses from 14 U.S. states, aged 25-44 years, completed a mailed questionnaire on their medical history and lifestyle characteristics [3]. Follow-up questionnaires have been sent every 2 years since. The active follow-up rate (number of person-years in the cohort, when participants are censored after their last questionnaire response) from 1989 to 2013 was $86 \%$ of the potential person-years. The study was approved by the local Institutional Review Board.

In all questionnaires, participants were asked to report any physician-diagnosed condition(s) that they had ever experienced (baseline) or with which they were diagnosed since the last questionnaire cycle (follow-up). Although no specific question on sarcoidosis was included, participants were asked to report "other major illness" in a final free-text field. We used this information to identify cases of physician-diagnosed sarcoidosis from 1989-2013. Although cases have not been validated by medical records, the validity of these nurses' health outcomes reports is generally $>80 \%$ for other conditions [12]. Furthermore, a 96\% agreement between self-report of sarcoidosis diagnosis and physician's report has been reported in the Black Women's Health Study [9].

Current height and weight, and weight at age 18 years were reported on the baseline questionnaire. Current weight was updated in all subsequent biennial questionnaires. Race (white, black, others), smoking status (never, ex- or current smoker) and pack-years (continuous), U.S. region (West, Midwest, South, Northeast), household income (>\$4 500 versus $\leqslant \$ 44500$ ), husband's education (high school or less, college graduate, graduate degree), physical activity $(<3,3-8.9,9-17.9,18-26.9,27-41.9, \geqslant 42$ metabolic equivalents (METs) per week) and alcohol consumption $(0,0.01-5,5.01-10,>10 \mathrm{~g} /$ day) were examined as potential confounders $[9,10]$. Associations of current BMI (i.e., BMI at the questionnaire cycle before

@ERSpublications

A higher BMI is prospectively associated with an increased risk of developing sarcoidosis in women http://ow.ly/OQ6k30f7XaQ

Cite this article as: Dumas O, Boggs KM, Cozier YC, et al. Prospective study of body mass index and risk of sarcoidosis in US women. Eur Respir J 2017; 50: 1701397 [https://doi.org/10.1183/13993003.013972017]. 
diagnosis), BMI at age 18 years, and weight change since age 18 years, with sarcoidosis incidence were evaluated using Cox proportional hazard models. All Cox models were stratified by age in months and calendar year. A two-sided $\mathrm{p}<0.05$ was considered statistically significant. All analyses were run using the SAS V.9 software (SAS Institute, Cary, NC, USA).

The study population consisted of 116312 women, without a history of sarcoidosis at baseline. At baseline, participants were on average 34 years old; $96 \%$ were white, $14 \%$ were current smokers and $21 \%$ were ex-smokers. The BMI at baseline was $<20 \mathrm{~kg} \cdot \mathrm{m}^{-2}$ in $15 \%$ of the participants; 20 to $24.9 \mathrm{~kg} \cdot \mathrm{m}^{-2}$ in $54 \%$; 25 to $29.9 \mathrm{~kg} \cdot \mathrm{m}^{-2}$ (overweight) in $19 \%$; and $\geqslant 30 \mathrm{~kg} \cdot \mathrm{m}^{-2}$ (obesity) in $12 \%$.

During 2457150 person-years of follow-up, from 1989 to 2013, 270 incident cases were reported. Higher current BMI was associated with increased risk of developing sarcoidosis during follow-up ( $\mathrm{p}$-trend $<0.001$, table 1). Similar results were observed in multivariable-adjusted models ( $\mathrm{p}$-trend $<0.001$ ), with significant associations in both overweight (hazard ratio (HR) 1.53, 95\% CI 1.12-2.10) and obese (HR 1.74, 95\% CI 1.26-2.40) women. Results were confirmed in a sensitivity analysis, in which sarcoidosis cases were restricted to participants who specifically indicated that the diagnosis occurred within 2 years before the questionnaire cycle during which they first reported sarcoidosis $(n=174)$. Stronger associations were found for the risk of sarcoidosis incidence in overweight (HR 1.75, 95\% CI 1.18-2.59) and obese (1.94, 1.302.91) women.

To rule out potential reverse causation (i.e., symptoms of sarcoidosis before diagnosis affecting weight), we examined the association between BMI at age 18 years and sarcoidosis incidence during follow-up. Although associations were attenuated compared to current BMI, a significant positive trend was observed between BMI at age 18 years and sarcoidosis incidence ( $\mathrm{p}$-trend=0.009, table 1 ). Higher weight gain since age 18 years was also associated with increased risk of sarcoidosis incidence $(p$-trend $=0.02)$.

TABLE 1 Prospective association between body mass index (BMI) and sarcoidosis incidence in women in the Nurses' Health Study II [3]

\section{Person-years Cases n Age-adjusted HR Multivariable-adjusted HR} (95\% Cl) $\quad(95 \% \mathrm{Cl})$

\begin{tabular}{|c|c|c|c|c|}
\hline \multicolumn{5}{|c|}{ Current $^{\#}$ BMI $\mathrm{kg} \cdot \mathrm{m}^{-2}$} \\
\hline$<20$ & 196733 & 15 & $1.00(0.58-1.74)$ & $1.02(0.59-1.78)$ \\
\hline $20-24.9$ (ref.) & 1042052 & 82 & 1 & 1 \\
\hline $25-29.9$ & 618960 & 80 & $1.65(1.21-2.26)$ & $1.53(1.12-2.10)$ \\
\hline$\geqslant 30$ & 526901 & 85 & $2.02(1.49-2.75)$ & $1.74(1.26-2.40)$ \\
\hline$p$-value & & & 0.001 & 0.001 \\
\hline \multicolumn{5}{|c|}{$\mathrm{BMI}$ at 18 years of age $\mathrm{kg} \cdot \mathrm{m}^{-2}$} \\
\hline$<20$ & 959879 & 88 & $0.81(0.62-1.06)$ & $0.82(0.63-1.07)$ \\
\hline $20-24.9$ (ref.) & 1226325 & 139 & 1 & 1 \\
\hline $25-29.9$ & 188343 & 29 & $1.38(0.92-2.06)$ & $1.31(0.87-1.95)$ \\
\hline$\geqslant 30$ & 58877 & 11 & $1.67(0.90-3.08)$ & $1.50(0.80-2.79)$ \\
\hline$p$-value & & & 0.002 & 0.009 \\
\hline \multicolumn{5}{|c|}{ Weight change since 18 years of age ${ }^{\pi}$ lb } \\
\hline$<-5$ & 132685 & 16 & $1.34(0.68-2.66)$ & $1.36(0.69-2.71)$ \\
\hline$-4.9-4.9$ (ref) & 229372 & 18 & 1 & 1 \\
\hline $5-14.9$ & 403705 & 24 & $0.78(0.42-1.44)$ & $0.76(0.41-1.40)$ \\
\hline $15-24.9$ & 401392 & 46 & $1.51(0.87-2.61)$ & $1.43(0.83-2.48)$ \\
\hline $25-34.9$ & 324565 & 33 & $1.31(0.73-2.33)$ & $1.20(0.67-2.14)$ \\
\hline $35-44.9$ & 246729 & 26 & $1.33(0.72-2.43)$ & $1.19(0.65-2.19)$ \\
\hline $45-54.9$ & 182979 & 24 & $1.64(0.88-3.04)$ & $1.44(0.77-2.68)$ \\
\hline$\geqslant 55$ & 447156 & 74 & $1.97(1.16-3.33)$ & $1.63(0.96-2.79)$ \\
\hline$p$-value & & & 0.001 & 0.02 \\
\hline
\end{tabular}

Multivariable models were adjusted for: age; race (white, black, other); smoking status (never-, ex- or current smoker) and pack-years (continuous); US region (West, Midwest, South, Northeast); household income (>\$44500 versus $\leqslant \$ 44500$, evaluated in 2001); husband's education (high school or less, college graduate, graduate degree, evaluated in 1999); physical activity $(<3,3-8.9,9-17.9,18-26.9,27-41.9, \geqslant 42$ metabolic equivalents (METs) per week); and alcohol consumption (0, 0.01-5, 5.01-10, >10 g per day). HR: hazard ratio. \#: at the questionnaire cycle before time of diagnosis. ?: analyses further adjusted for BMI at 18 years of age. Observations with missing values for smoking status or region $(<0.5 \%)$ were excluded from analyses (multivariable-adjusted models). For each other covariate with $>0.5 \%$ missing values, including current $\mathrm{BMI}(3.0 \%), \mathrm{BMI}$ at 18 years of age $(0.9 \%)$ or weight change since 18 years of age $(3.6 \%)$, a "missing" category was included in the model. 
In this large cohort of predominantly white U.S. women, increased risks of sarcoidosis were observed in both overweight and obese participants. Consistent findings have been reported in a Danish register-based study examining the association between pre-pregnancy BMI in women and the development of several disorders, including sarcoidosis [10]. Similar results have also been reported in a population of black women in the U.S. [9], but only for those with morbid obesity $\left(\mathrm{BMI} \geqslant 35 \mathrm{~kg} \cdot \mathrm{m}^{-2}\right)$.

Chronic systemic inflammation associated with adiposity is suspected to have a role in the development of many disorders, including respiratory [8] and autoimmune diseases [10]. Adipokines produced in the obese adipose tissue have broad immunomodulatory effects. In particular, they could induce a proinflammatory process in the lungs $[8,9]$, which is involved in most sarcoidosis cases. Furthermore, some investigators have suggested that the exaggerated inflammatory response to a causal antigen, leading to the formation of granulomas in sarcoidosis might occur, or be enhanced, in an inflammatory environment [13]. Our results are consistent with the hypothesis that adiposity-related inflammation could contribute to the development of sarcoidosis.

In summary, this analysis of a large cohort of U.S. women followed-up over 24 years showed that higher BMI and weight gain was prospectively associated with a higher risk of incident sarcoidosis. Given the elevated prevalence of overweight and obesity in the U.S. and other developed countries, and limited understanding of sarcoidosis aetiology, the potential impact of adiposity-related inflammation merits further investigation.

Orianne Dumas $\varpi^{1,2}$, Krislyn M. Boggs ${ }^{3}$, Yvette C. Cozier ${ }^{4}$, Meir J. Stampfer ${ }^{3}$ and Carlos A. Camargo Jr ${ }^{3,5}$

${ }^{1}$ INSERM U1168, VIMA: Aging and Chronic Diseases, Epidemiological and Public Health Approaches, F-94807, Villejuif, France. ${ }^{2}$ Université de Versailles St-Quentin-en-Yvelines, UMR-S 1168, F-78180, Montigny le Bretonneux, France. ${ }^{3}$ Channing Division of Network Medicine, Dept of Medicine, Brigham and Women's Hospital and Harvard Medical School, Boston, MA, USA. ${ }^{4}$ Slone Epidemiology Center, Boston University, Boston, MA, USA. ${ }^{5}$ Dept of Emergency Medicine, Massachusetts General Hospital, Harvard Medical School, Boston, MA, USA.

Correspondence: Orianne Dumas, INSERM UMRS 1168, VIMA - Aging and Chronic Diseases, Epidemiological and Public Health Approaches, 16, avenue Paul Vaillant Couturier, 94807 Villejuif cedex, France.

E-mail: orianne.dumas@inserm.fr

Received: July 122017 | Accepted: July 172017

Support statement: Funding was provided by the National Institutes of Health (UM1 CA176726). Funding information for this article has been deposited with the Crossref Funding Registry.

Conflict of interest: None declared.

Acknowledgements: The Nurses' Health Study II is coordinated at the Channing Division of Network Medicine, Brigham and Women's Hospital, Boston, MA. We would like to thank the participants and staff of the Nurses' Health Study II for their valuable contributions.

\section{References}

Valeyre D, Prasse A, Nunes H, et al. Sarcoidosis. Lancet 2014; 383: 1155-1167.

Gerke AK. Morbidity and mortality in sarcoidosis. Curr Opin Pulm Med 2014; 20: 472-478.

Dumas O, Abramovitz L, Wiley AS, et al. Epidemiology of sarcoidosis in a prospective cohort study of U.S. women. Ann Am Thorac Soc 2016; 13: 67-71.

4 Jamilloux Y, Maucort-Boulch D, Kerever S, et al. Sarcoidosis-related mortality in France: a multiple-cause-of-death analysis. Eur Respir J 2016; 48: 1700-1709.

5 Arkema EV, Grunewald J, Kullberg S, et al. Sarcoidosis incidence and prevalence: a nationwide register-based assessment in Sweden. Eur Respir J 2016; 7: 113-118.

6 Newman LS, Rose CS, Bresnitz EA, et al. A case control etiologic study of sarcoidosis: environmental and occupational risk factors. Am J Respir Crit Care Med 2004; 170: 1324-1330.

7 Liu H, Patel D, Welch AM, et al. Association between occupational exposures and sarcoidosis: an analysis from death certificates in the United States, 1988-1999. Chest 2016; 150: 289-298.

$8 \quad$ Umetsu DT. Mechanisms by which obesity impacts asthma. Thorax 2017; 72: 174-177.

9 Cozier YC, Coogan PF, Govender P, et al. Obesity and weight gain in relation to incidence of sarcoidosis in US black women. Chest 2015; 147: 1086-1093.

10 Harpsøe MC, Basit S, Andersson M, et al. Body mass index and risk of autoimmune diseases: a study within the Danish National Birth Cohort. Int J Epidemiol 2014; 43: 843-855.

11 Ungprasert P, Crowson CS, Matteson EL. Smoking, obesity and risk of sarcoidosis: a population-based nested case-control study. Respir Med 2016; 120: 87-90.

12 Colditz GA, Martin P, Stampfer MJ, et al. Validation of questionnaire information on risk factors and disease outcomes in a prospective cohort study of women. Am J Epidemiol 1986; 123: 894-900.

13 Newman KL, Newman LS. Occupational causes of sarcoidosis. Curr Opin Allergy Clin Immunol 2012; 12 $145-150$. 